\title{
Autosomal dominant retinitis pigmentosa: a log quotient analysis of the photopic and scotopic b-wave amplitude
}

\author{
HIROYUKI IIJIMA, ${ }^{2}$ DEIDRE A MARTIN, ${ }^{\prime}$ AND JOHN R HECKENLIVELY \\ From the 'Jules Stein Eye Institute and Department of Ophthalmology, UCLA School of Medicine, Los \\ Angeles, California, USA, and the ${ }^{2}$ Department of Ophthalmology, Yamanashi Medical College, Tamaho, \\ Yamanashi, Japan
}

SUMmaRY The relationship of the photopic and the scotopic b-wave amplitudes of the electroretinogram was studied in 85 normal subjects and 25 patients with autosomal dominant retinitis pigmentosa, in which one amplitude was at least 20 microvolts. The log quotient of their b-wave amplitudes - that is log of the photopic b-wave amplitude divided by the scotopic b-wave amplitude - was considered to represent the activity of cones relative to rods. The log quotient values had a normal gaussian distribution in the normal control eyes, while they formed two groups in the patients with autosomal dominant retinitis pigmentosa. In the first group (type 1), the scotopic b-wave was non-recordable while the photopic b-wave amplitude was larger than 20 microvolts in all cases, indicating that the log quotient is larger than 0.5 and that the rod system is much more severely affected than the cone system. The second group (type 2 ) had a log quotient smaller than 0.5 and its distribution almost overlapped the normal one, indicating more symmetrical damage in the cone and rod systems. The mean final rod threshold at 45 minutes for type 1 was significantly higher than that for type 2 . The log quotient proved to be a useful index for analysing the cone and rod involvement and consequently provides a better understanding of autosomal dominant retinitis pigmentosa.

Retinitis pigmentosa (RP) is a group of disorders that are characterised by progressive visual field loss resulting from a degenerative process of the photoreceptor and pigment epithelial cell layers. Classification by genetic pattern is generally accepted. ${ }^{1}$ However, it has been suggested that even within the same genetic pattern a heterogeneous disease process may exist. ${ }^{2}$ Among genetic patterns autosomal dominant retinitis pigmentosa (ADRP) has been intensively studied by several investigators. ${ }^{3-6}$ Their classifications, though determined by different examinations, are based on cone and rod involvement in the disease process and agree broadly on the point that ADRP (excluding sector RP) can be divided into two groups.

The cone and rod involvement in retinal disorders can be evaluated easily by amplitudes of the photopic

Correspondence to John R Heckenlively, MD, Jules Stein Eye Institute, UCLA Medical Center, Los Angeles, CA 90024-1771, USA. and scotopic electroretinogram (ERG), which has been a routine method for evaluating RP patients. ${ }^{17}$ In this study we propose the log quotient of the photopic b-wave amplitude (bp) divided by that of the scotopic b-wave (bs) - that is, log (bp/bs) - as a good indicator for assessing the cone and rod involvement in ADRP patients.

\section{Subjects and methods}

Eighty-five subjects ( 38 male and 47 female, aged 3 to 77 years) with normal ophthalmic findings were tested to study a normal distribution of the parameters. The mean (and standard deviation) of age for males and females were 31 (17) and 34 (20) years respectively, which were not significantly different.

For ADRP we selected 25 patients with recordable ERGs who had either photopic or scotopic ERG amplitudes larger than $20 \mu \mathrm{V}$ with single flash techniques from a total of 89 consecutive ADRP 
Table 1 Summary of clinical findings

\begin{tabular}{|c|c|c|c|c|c|c|c|c|c|}
\hline Case & $\begin{array}{l}\text { Agel } \\
\text { Sex }\end{array}$ & $\begin{array}{l}\text { Onset } \\
\text { of } N B\end{array}$ & $\begin{array}{l}\text { Onset } \\
\text { of VFL }\end{array}$ & Acui & $\begin{array}{l}b p \\
(\mu V)\end{array}$ & $\begin{array}{l}b s \\
(\mu V)\end{array}$ & $\begin{array}{l}\log \\
(b p / b s)\end{array}$ & $\begin{array}{l}b p t \\
(m s)\end{array}$ & $D A$ \\
\hline \multicolumn{10}{|c|}{ Type 1} \\
\hline 415 & $16 / F$ & 15 & UA & $20 / 25$ & 220 & $0^{-}$ & & 36 & 1.0 \\
\hline 416 & $24 / F$ & 1 & 24 & $20 / 20$ & 80 & 0 & & 44 & $4 \cdot 2$ \\
\hline 634 & $45 / F$ & 14 & UA & $20 / 20$ & 20 & 0 & & 44 & $4 \cdot 35$ \\
\hline 823 & $23 / \mathrm{M}$ & UA & UA & $20 / 20$ & 40 & 0 & & 32 & 1.0 \\
\hline 734 & $27 / M$ & 15 & 15 & $20 / 20$ & 50 & 0 & & 41 & $2 \cdot 4$ \\
\hline 385 & $41 / \mathrm{M}$ & 31 & 34 & CF & 20 & 0 & $>0.5$ & 54 & $4 \cdot 8$ \\
\hline 849 & $40 / \mathrm{M}$ & 34 & 34 & $20 / 25$ & 90 & 0 & & 44 & $5 \cdot 0$ \\
\hline 355 & $44 / M$ & UA & UA & $20 / 20$ & 30 & 0 & & 48 & $1 \cdot 15$ \\
\hline 417 & $20 / F$ & 1 & 17 & $20 / 30$ & 70 & 0 & & 46 & $4 \cdot 5$ \\
\hline 646 & $19 / \mathrm{M}$ & 8 & 16 & $20 / 25$ & 100 & 0 & & 46 & $2 \cdot 4$ \\
\hline 86 & $32 / F$ & 24 & UA & $20 / 20$ & 40 & 0 & & 44 & $3 \cdot 35$ \\
\hline 676 & $29 / \mathrm{M}$ & UA & UA & $20 / 30$ & 50 & 0 & & 48 & 0.6 \\
\hline \multicolumn{10}{|c|}{ Type 2} \\
\hline 1023 & $37 / F$ & 32 & 32 & $20 / 30$ & 40 & 20 & $0 \cdot 301$ & 42 & $2 \cdot 4$ \\
\hline 644 & $50 / \mathrm{M}$ & 30 & 30 & $20 / 20$ & 110 & 80 & $0 \cdot 138$ & 32 & $3 \cdot 15$ \\
\hline 754 & $31 / \mathrm{M}$ & 21 & UA & $20 / 30$ & 60 & 60 & 0 & 42 & 3.55 \\
\hline 142 & $38 / \mathrm{F}$ & 38 & UA & $20 / 20$ & 150 & 160 & -0.028 & 32 & $1 \cdot 15$ \\
\hline 483 & $34 / F$ & UA & UA & $20 / 20$ & 180 & 200 & -0.046 & 34 & ND \\
\hline 950 & $13 / F$ & 12 & 12 & $20 / 50$ & 60 & 90 & -0 & 40 & ND \\
\hline 263 & $13 / \mathrm{F}$ & 13 & 13 & $20 / 25$ & 30 & 50 & -0.222 & 40 & 0.5 \\
\hline 523 & $34 / F$ & UA & UA & $20 / 30$ & 50 & 110 & $-0 \cdot 301$ & 36 & $0 \cdot 2$ \\
\hline 949 & $45 / F$ & 36 & 39 & $20 / 200$ & 50 & 100 & -0.301 & 42 & $2 \cdot 0$ \\
\hline 1028 & $15 / \mathrm{M}$ & UA & UA & $20 / 20$ & 120 & 250 & -0.319 & 32 & $1 \cdot 0$ \\
\hline 1025 & $21 / \mathrm{M}$ & 21 & 21 & $20 / 20$ & 70 & 170 & -0.385 & 35 & $0 \cdot 5$ \\
\hline 1024 & $41 / M$ & 39 & 39 & $20 / 25$ & 70 & 200 & -0.456 & 34 & $2 \cdot 1$ \\
\hline 468 & 29/M & 9 & 24 & $20 / 25$ & 10 & 50 & -0.699 & 36 & 0.7 \\
\hline
\end{tabular}

NB: night blindness; VFL: visual field loss; bp: photopic b-wave amplitude; bs: scotopic b-wave amplitude; bpt: photopic b-wave implicit time; DA: final threshold of dark adaptation; CF: count fingers; UA: unaware of the symptom; ND: the test was not done.

patients in the UCLA RP Registry. The clinical findings are listed in Table 1.

Full-field (Ganzfeld) ERGs with Burian-Allen contact lens electrode were recorded as described previously. ${ }^{8}$ The scotopic ERG was recorded with a $\mathrm{dim}$ white flash $\left(-1.58 \mathrm{log} \mathrm{cd} / \mathrm{m}^{2} / \mathrm{s}\right)$ obtained by I-8 on Grass photostimulator (PS-22) and Kodak Wratten filter ND 2.0 after dark adaptation for 30 minutes or more. The photopic ERG with I-16 white flash $(0.85$ $\log \mathrm{cd} / \mathrm{m}^{2} / \mathrm{s}$ ) was recorded in the presence of a steady $27.4 \mathrm{~cd} / \mathrm{m}^{2}$ background light. This method allowed recordings of $5 \mu \mathrm{V}$ or more to be measured. Since responses from both eyes were symmetrical with few exceptions both in normal persons and RP patients, responses from the right eye of each subject were analysed except normal controls with retinal disease in the right eye.

The final threshold of dark adaptation was measured after 45 minutes of dark adaptation by Goldmann-Weekers adaptometer with a $1^{\circ}$ test field located $12^{\circ}$ below the red fixation light.

\section{Results}

The distributions of $b p / b s$ and the $\log (b p / b s)$ of
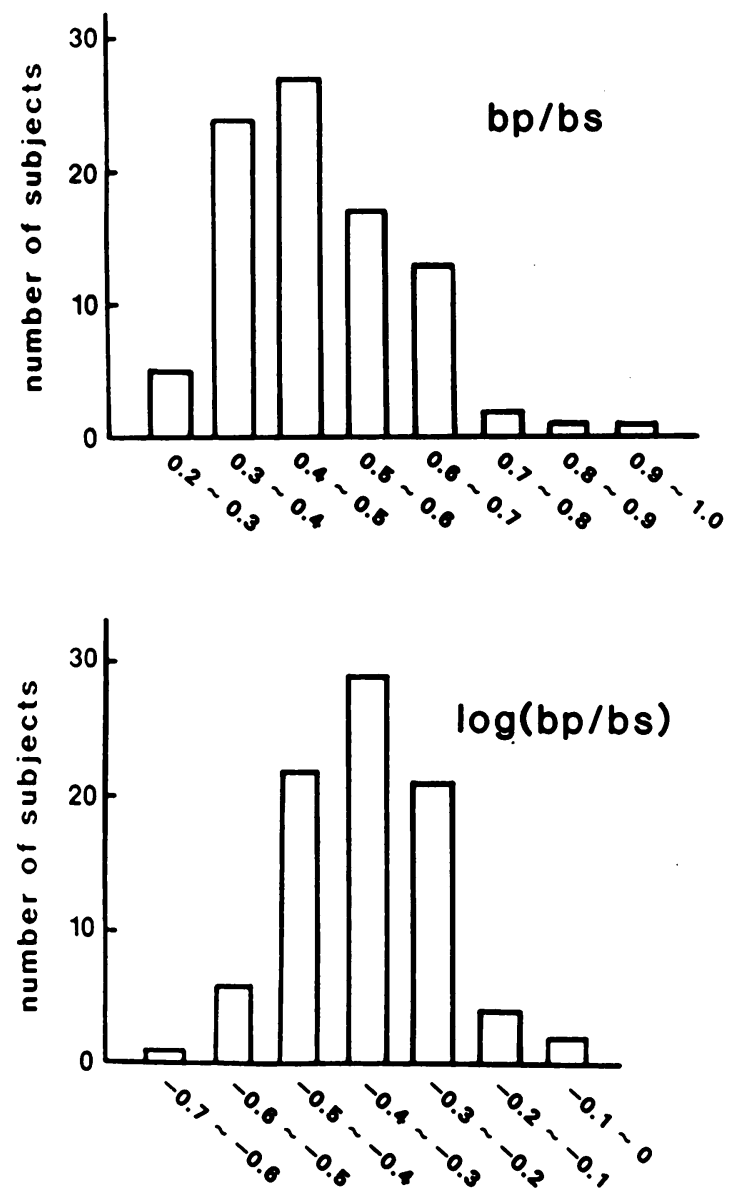

Fig. 1 Histograms for bp/bs and log(bp/bs) of 85 normal control subjects. $-0 \cdot 7 \sim$ means the individual $\log (\mathrm{bp} / \mathrm{bs})$ fell within the range -0.7 to $<0.06$.

normal control eyes are shown in Fig. 1. The histogram of bp/bs is asymmetrical, with its peak deviating towards the lower end, while the histogram of $\log (\mathrm{bp} / \mathrm{bs})$ appears almost symmetrical. The cumulative percentage frequencies were plotted on probability paper in Fig. 2. The line for bp/bs curves up in the middle, while the line for $\log (\mathrm{bp} / \mathrm{bs})$ appears almost straight, indicating that $\log (\mathrm{bp} / \mathrm{bs})$ follows the normal gaussian distribution with a mean of -0.35 and a standard deviation of $0 \cdot 11$. There was no statistical difference between the values of $\log (\mathrm{bp} / \mathrm{bs})$ in male and female (male: $-0.33 \pm 0.12$, female: $-0 \cdot 36 \pm 0 \cdot 11)$. The scattergram of $\log (\mathrm{bp} / \mathrm{bs})$ against age shown in Fig. 3 demonstrates no significant age effect (correlation coefficient -0.085 ).

In patients with ADRP, the $\log (\mathrm{bp} / \mathrm{bs})$ for 13 patients out of 25 was distributed between -0.75 and $0 \cdot 5$, forming a single peak between -0.5 and -0.25 


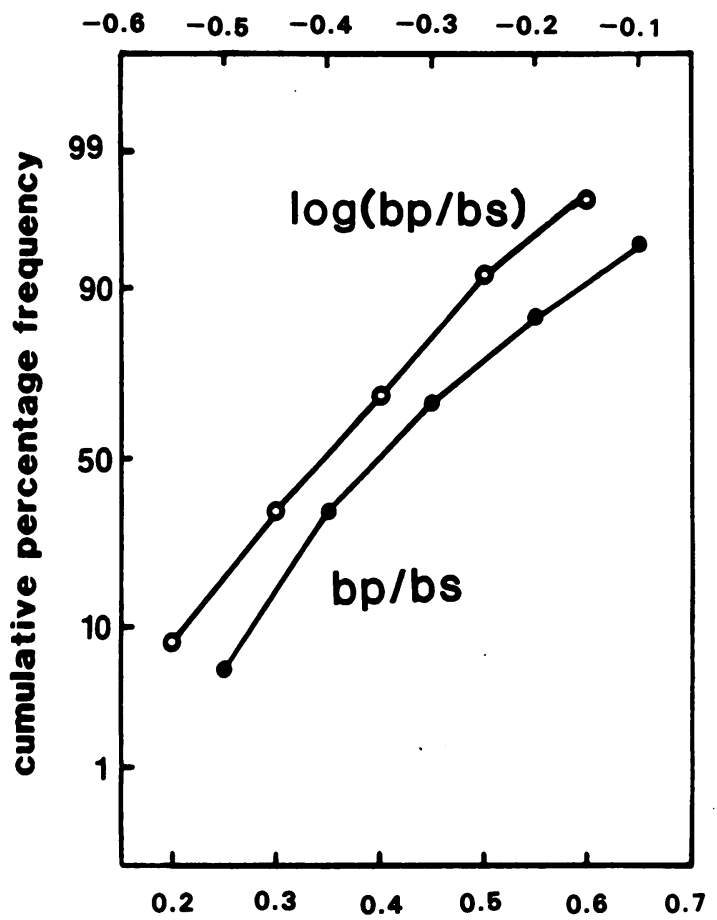

Fig. 2 The cumulative percentage frequencies of the results in Fig. 1 expressed on a probability paper. The closed circles are for bp/bs; their values are shown at the bottom. The open circles are for $\log (b p / b s)$; their values are shown at the top.
(Fig. 4). Although the 12 remaining patients showed unrecordable scotopic ERGs and thus their $\log (\mathrm{bp} / \mathrm{bs})$ is unavailable, it could be estimated as larger than 0.5 because their unrecordable scotopic b-wave actually means less than $5 \mu \mathrm{V}$ and the photopic b-wave in all cases was larger than $20 \mu \mathrm{V}$. Thus according to the value of $\log (\mathrm{bp} / \mathrm{bs})$ all patients could be classified into two groups, namely, type 1 and type 2, with an arbitrary limit of $0 \cdot 5$. A summary of clinical findings of both groups is given in Table 2 . Type 1 had higher final dark adaptation threshold values $(p \leqslant 0.05)$ and a more delayed photopic $b$-wave implicit time $(p \leqslant 0 \cdot 01)$. More than one patient from each of three families were included in this study. Family No. 1 are cases 415, 416, and 417, family No. 2 cases $1023,1024,1025,1028$, and family No. 3 cases 949 and 950 . All members of each family belonged to the same type.

\section{Discussion}

Cone and rod functions are evaluated by the photopic and scotopic ERGs respectively, and their amplitudes help the analysis of cone versus rod involvement in photoreceptor disease. ${ }^{17}$ In general rod-cone and cone-rod degeneration are diagnosed on the basis of which system is predominantly involved. ${ }^{910}$ When either the photopic or scotopic ERG is disturbed while the other shows a good response, the relative involvement of rods and cones is easily understood. But there have been no

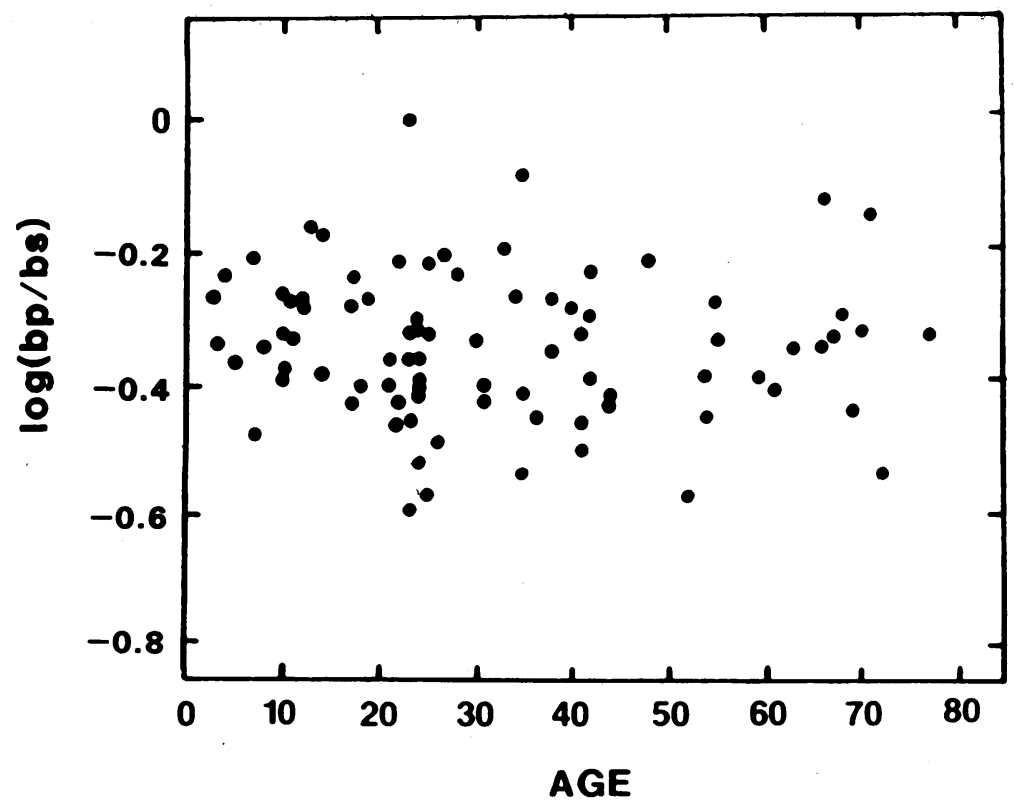

Fig. 3 Log(bp/bs) versus age in 85 normal controls. 


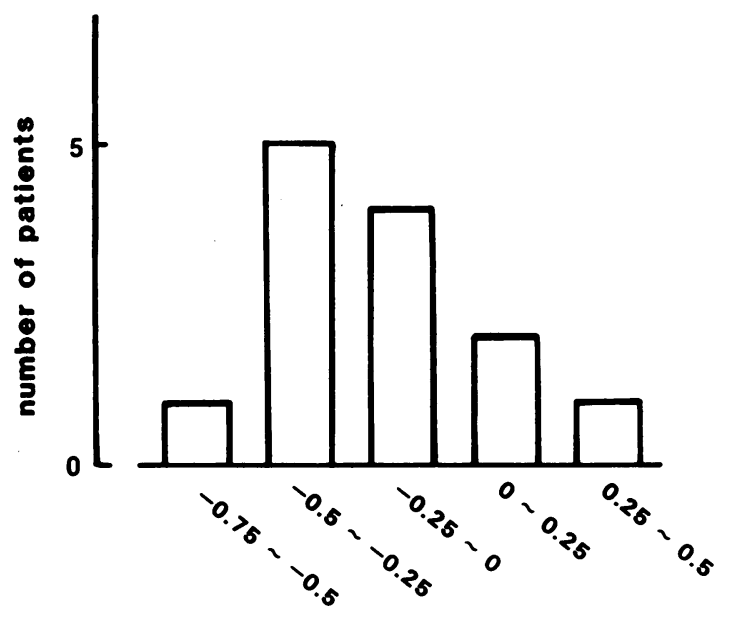

$\log (\mathrm{bp} / \mathrm{bs})$

Fig. 4 Histogram for $\log (\mathrm{bp} / \mathrm{bs})$ in patients with type 2 autosomal dominant retinitis pigmentosa.

reasonable criteria for determining predominant involvement of cones or rods when both photopic and scotopic b-wave amplitudes are decreased. We proposed a parameter of $\mathrm{bp} / \mathrm{bs}$ for that purpose because it represents relative activity of cones compared with that of rods. A similar concept was introduced by Birch and Fish, ${ }^{11}$ who recorded cone and rod responses with scotopically matched blue and red filters so that both amplitudes are equal in normal eyes, and defined cone-rod degeneration as having the quotient of rod/cone amplitude more than $1 \cdot 0$. But there was no reasonable basis to set a borderline at 1.0 and to subdivide RP patients at that point because there was no information about the distribution of this index in either the normal population of RP patients. Therefore we studied the distribution of $\mathrm{bp} / \mathrm{bs}$ in normal eyes and ADRP patients.

Since normal eyes showed log normal distribution, $\log (\mathrm{bp} / \mathrm{bs})$ is considered as a more reasonable parameter rather than $\mathrm{bp} / \mathrm{bs}$. The results from normal

Table 2 Clinical findings for type 1 and type 2

\begin{tabular}{lcc}
\hline & Type 1 & Type 2 \\
\hline Number of patients & 12 & 13 \\
Age & $30(10)$ & $31(12)$ \\
Age at onset of night blindness & $16(11)$ & $25(11)$ \\
Photopic b-wave amplitude & $68(56)$ & $77(49)$ \\
Scotopic b-wave amplitude & 0 & $118(71)$ \\
Log(bp/bs) & $<0 \cdot 5$ & $-0 \cdot 19(0 \cdot 26)$ \\
Photopic b-wave implicit time & $43.9(5 \cdot 7)$ & $36 \cdot 7(4 \cdot 0)^{* *}$ \\
Final threshold of dark adaptation & $2 \cdot 9(1 \cdot 7)$ & $1 \cdot 6(1 \cdot 1)^{*}$ \\
\hline
\end{tabular}

Values are means (SD). ${ }^{*} \mathrm{p}<0.05,{ }^{* *} \mathrm{p}<0.01$. eyes also showed that $\log (\mathrm{bp} / \mathrm{bs})$ neither differs significantly between male and female nor is correlated with age. These features are different from other ERG parameters such as amplitudes and implicit times, which are reported to be sex and or age dependent ${ }^{12-14}$ and are thought to be advantageous as clinical parameters.

The distribution pattern of $\log (\mathrm{bp} / \mathrm{bs})$ in ADRP patients in this study clearly showed that ADRP patients can be divided into at least two subgroups, type 1 and type 2 . Since the distribution in type 2 nearly overlaps the normal distribution, it could be said that their cones and rods are equally damaged, whereas in type 1 the rods are more extensively damaged.

Type 1 and type 2 were different in the degree of final threshold elevation and in the delay of the photopic b-wave implicit time, with greater delays in type 1 . The difference in age at onset of night blindness between the groups was not significant, which might be owing to the relatively small number of subjects. This finding is in marked contrast to the results of Lyness et al.,$^{5}$ where age of onset correlated with type. Their selection of types was based on psychophysical testing, while our group is based on electrophysiological results. Since data for age at onset represent subjective reporting by the patient and are influenced by variant living conditions, differences in age of onset are not surprising. The lack of intrafamilial variation is strong evidence in support of our classification.

There have been several reports on RP subclassification. Marmor ${ }^{15}$ showed that RP patients with normal cone implicit time have a better visual prognosis than those with prolonged cone implicit time irrespective of their inheritance pattern, and called this group of patients 'delimited RP'. Type 2 patients in our classification had significantly less delay in photopic b-wave implicit times than type 1 patients. Since cone implicit times are related to amplitudes of the photopic b-wave and the scotopic b-wave, ${ }^{16}$ which are subject to progression of the disease process, it is not certain whether cone b-wave implicit times can render a substantial subclassification of RP types, and good serial ERG studies will need to be performed in the delimited RP group to substantiate this point.

Arden and colleagues ${ }^{3}$ defined two subgroups in ADRP based solely on the presence or absence of the scotopic ERG. Their subgroup A, in which no scotopic ERG was evoked, broadly (though not exactly) corresponds to our type 1 , and their subgroup $B$, in which the scotopic b-wave is present, to our type 2. Considering the progressive nature of ADRP, patients in their subgroup A could have had recordable scotopic ERGs in early stages, and those 
in subgroup B should lose the scotopic ERG in a very advanced stage. This problem may have also influenced our study, though the methodology helped to minimise it.

There have been attempts to subclassify ADRP by psychophysical techniques. Massof and Finkelstein ${ }^{4}$ defined two types of ADRP-type 1 with early diffuse loss of rod sensitivity and later loss of cone sensitivity, and type 2 with a regionalised and combined loss of rod and cone sensitivity. Lyness and associates $^{5}$ proposed a similar subclassification on the basis of a two-colour automated static perimeter. In their subgroup D severe and diffuse loss of rod function is shown with patchy loss of cone function. In their subgroup $\mathbf{R}$ loss of rod function is regional and accompanied by loss of cone function.

These psychophysical methods for subclassification are reasonable, but unfortunately the methods have not become a part of the routine clinical examination, and standardisation among research centres is not easy. Our classification by $\log (\mathrm{bp} / \mathrm{bs})$ is an objective method and can be used wherever electroretinographic studies of RP patients are performed in which the cone and rod systems are measured separately.

\section{References}

1 Marmor MF, Aguirre G, Arden G, et al. Retinitis pigmentosa. A symposium on terminology and methods of examination. Ophthalmology 1983; 90: 126-31.

2 Massof RW. Psychophysical subclassification of retinitis pigmentosa. In: LaVail MM, Hollyfield JG, Anderson RE, eds. Retinal degeneration: experimental and clinical studies. New York: Liss, 1985: 91-107.
3 Arden GB, Carter RM, Hogg CR, et al. Rod and cone activity in patients with dominantly inherited retinitis pigmentosa: comparisons between psychophysical and electroretinographic measurements. Br J Ophthalmol 1983; 67: 405-18.

4 Massof RW, Finkelstein D. Two forms of autosomal dominant primary retinitis pigmentosa. Doc Ophthalmol 1981; 51: 289346.

5 Lyness AL, Ernst W, Quinlan MP, et al. A clinical, psychophysical, and electroretinographic survey of patients with autosomal dominant retinitis pigmentosa. Br J Ophthalmol 1985; 69: 326-39.

6 Fishman GA, Alexander KR, Anderson RJ. Autosomal dominant retinitis pigmentosa. A method of classification. Arch Ophthalmol 1985; 103: 366-74.

7 Heckenlively JR. The diagnosis and classification of retinitis pigmentosa. In: Heckenlively $\mathrm{JH}$, ed. Retinitis pigmentosa. Philadelphia: Lippincott, 1988: 6-14.

8 Heckenlively JR. RP cone-rod degeneration. Trans Am Ophthalmol Soc 1987; 85: 438-70.

9 Krill AE. Rod-cone dystrophies. In: Krill AE, Archer DB, eds. Krill's hereditary retinal and choroidal diseases. Hagerstown: Harper and Row, 1977: 479-644.

10 Berson EL, Gouras P, Gunkel RD. Progressive cone-rod degeneration. Arch Ophthalmol 1968; 80: 68-76.

11 Birch DG, Fish GE. Rod ERGs in retinitis pigmentosa and conerod degeneration. Invest Ophthalmol Vis Sci 1987; 28: 140-50.

12 Martin DA, Heckenlively JR. The normal electroretinogram. Doc Ophthalmol Proc Ser 1982; 31: 135-44.

13 Weleber RG. The effect of age on human cone and rod ganzfeld electroretinograms. Invest Ophthalmol Vis Sci 1981; 20: 392-9.

14 Wright CE, Williams DE, Drasdo N, Harding GFA. The influence of age on the electroretinogram and visual evoked potential. Doc Ophthalmol 1985; 59: 365-84.

15 Marmor MF. The electroretinogram in retinitis pigmentosa. Arch Ophthalmol 1979; 97: 1300-4.

16 Birch DG, Sandberg MA. Dependence of cone b-wave implicit time on rod amplitude in retinitis pigmentosa. Vision Res 1987; 27: $1105-12$.

Accepted for publication 18 July 1988. 